\section{Energieangebotspolitik war gestern}

\author{
von Ernst Ulrich von Weizsäcker, \\ Emmendingen
}

\section{Bislang: Energieangebotspolitik}

Seit Jahrtausenden war das Auffinden und Nutzen von Energie eine der wichtigsten Voraussetzungen für menschliches Überleben und später für das wirtschaftliche Wachstum. Soweit es so etwas wie Energiepolitik gab, war diese seit alters her Energieangebotspolitik. Man „erschloss“ neue Quellen durch Abholzen, durch Wind- und Wassermühlen, später durch Kohleabbau, Ö1und Gaspumpen, Nutzung der Atomkernspaltung und den Ausbau der erneuerbaren Energiequellen. Die Zunahme des wirtschaftlich messbaren Wohlstands war und blieb eng verknüpft mit dem Energieverbrauch.

In ebenso selbstverständlicher wie naiver Verlängerung dieser Politik dreht sich die heutige Energiediskussion in der Hauptsache um die Bereitstellung von immer mehr Energie. Man diagnostiziert immer neue „Lücken“ in der Bedarfsdeckung. Der politische Streit geht fast ausschließlich um die Frage, welche Energieangebote ökologisch und ökonomisch am günstigsten sind. Im Rahmen dieses Streits schälen sich aus der heutigen Energiediskussion in Deutschland die folgenden vier Strategien heraus:

1. neue Kohlekraftwerke bauen und das dabei entstehende $\mathrm{CO}_{2}$ um des Klimaschutzes willen zu großen Teilen abfangen und unter der Erde vergraben („Carbon Capture and Storage", CCS (s. Beitrag Fischer et al. in diesem Schwerpunkt);

2. die Laufzeiten der Atomkraftwerke deutlich verlängern, oft in der nicht ausgesprochenen Hoffnung, später auch neue zu bauen;

3. viel Energie importieren;

4. ein äußert forcierter Ausbau der erneuerbaren Energien.

Jede dieser Strategien ist mit erheblichen Problemen verbunden, die eine intensive Beschäfti- gung rechtfertigen. Dies ist hier aber nicht meine Absicht.

\section{Das Energiekonzept der Bundesregierung vom September 2010}

Das Ende September 2010 von der Bundesregierung beschlossene Energiekonzept kombiniert die erste, zweite und vierte Strategie und versucht (vernünftigerweise), den Energieimport zu reduzieren. Bis 2050 sollen 60 Prozent des Energiebedarfs aus erneuerbaren Energien bestritten werden, wobei v. a. der Windenergie an Land und auf See große Erweiterungspotenziale zugemessen werden. Insbesondere in diesem Zusammenhang enthält das Energiekonzept auch die Absicht eines massiven Ausbaus der Stromnetze. Das macht allerdings nur Sinn, wenn diese Netze in der Hauptsache als raum- und umweltschonende Hochspannungs-Gleichstromübertragung geplant werden.

Auch die Energieeffizienz bekommt breiten Raum und wird als „Schlüsselfrage“ bezeichnet. Große Bedeutung hat dabei der Gebäudebereich, aber auch die Industrie soll ihre Effizienzpotenziale „ausschöpfen“.

In der öffentlichen Diskussion und Kritik am Energiekonzept steht v. a. die Laufzeitverlängerung der vorhandenen Kernkraftwerke. Hier ist in der Tat zu kritisieren, dass kleine Energieversorger besonders auf der Ebene der Stadtwerke, die sich eigenverantwortlich um die Erhöhung des Anteils erneuerbarer Energie gekümmert haben und die ehrgeizige Effizienzmaßnahmen ergriffen oder geplant haben, durch das Billigangebot von Strom aus abgeschriebenen Kernkraftwerken stark behindert, teilweise in ihrer Existenz gefährdet werden.

Was mir jedoch insgesamt viel zu konventionell erscheint, ist das (stillschweigende) Festhalten an der Idee, dass Energie möglichst billig angeboten werden müsse. So als ob die derzeitigen Preise von fünf Eurocent pro Kilowattstunde an der Leipziger Strombörse nicht schon ein Tiefschlag für ehrgeizigen Klimaschutz und Ressourcenschonung wären! Die Billig-Mentalität kommt auch in Sätzen wie diesem zum Ausdruck: „Um die erheblichen Potenziale zur Energie- und Stromeinsparung zu heben, sind 
viele Maßnahmen erforderlich, die langfristig die Energiekosten für Wirtschaft, Kommunen und Verbraucher senken ..." (BMWi 2010, S. 13) Und hierfür soll nun wieder ein Fonds eingerichtet werden, der die zu ergreifenden Maßnahmen subventioniert. Der Fortschritt besteht lediglich darin, dass wenigstens nicht mehr der Mehrverbrauch subventioniert werden soll.

\section{3 Ökologische Preispolitik}

Die durch die oben skizzierten vier Strategien charakterisierte konventionelle Politik wird auch dann in große Schwierigkeiten geraten, wenn die vier Strategien in ein optimiertes Mischungsverhältnis gebracht werden, wie das die Absicht des Energiekonzepts der Bundesregierung ist. Die öffentlich diskutierten Strategien der Opposition sind in dieser Hinsicht kaum besser, weil sie wie die Regierung dem Traum nachhängen, Energie dürfe, ja solle billig angeboten werden. Und dann kommt der Zusatztraum, dieses sei mit einem noch ehrgeizigeren Ausbau der erneuerbaren Energien auch erreichbar.

Ich stelle dieser wogenden Streitdiskussion eine prinzipielle Alternative entgegen. Diese beruht

- auf der Analyse, dass die Effizienzpotenziale überhaupt nicht statisch sind und ,ausgeschöpft" werden können, sondern dass sie vielmehr dynamisch sind und nahezu unbegrenzt erweitert werden können;

- auf der Überlegung, dass die Energieproduktivität sehr analog zur Arbeitsproduktivität im Gleichklang mit der Verteuerung der Energie mittelfristig um einen Faktor fünf, langfristig um einen Faktor 20 steigen könnte; dieses ist der Kerninhalt des neuen Buches „Faktor Fünf" (von Weizsäcker et al. 2010);

- auf der dann allerdings trivialen Feststellung, dass eine derart dramatische Erhöhung der Energieproduktivität ceteris paribus die heutigen Streitfragen über Klimaschutz und Kernenergie oder auch über Schäden durch Biosprit schlicht zum Verschwinden bringen würde.

Die Kernthese ist im zweiten Punkt versteckt: Die Energie müsste ,,im Gleichklang“" mit steigender Energieproduktivität laufend verteuert werden. Die immer ehrgeizigere Nutzung der Effizienzpotenziale würde also gerade nicht zu Kostenreduktionen beim Energieeinkauf führen, wohl aber zu gewaltigen Wohlfahrtsgewinnen an völlig anderen Stellen. Diese anderen Stellen sind nicht nur die Vermeidung großer Umweltkatastrophen und der Abhängigkeit von immer riskanteren Ölquellen, sondern auch eine steile, stetige Modernisierung mit einer anzunehmenden deutlichen Verbesserung der Beschäftigungslage. Die Verminderung der $\mathrm{CO}_{2}$-Intensität und der Energieintensität von Gebäuden, Industrie, Landwirtschaft und Verkehr geht ja einher mit einer Steigerung der Informationsintensität und einer durchgreifenden System-Modernisierung, wie dies in Teil I des genannten Buches „Faktor Fünf" ausgeführt wird.

Die Zentrierung der Energiepolitik bei der Erhöhung der Energieproduktivität widerspricht natürlich auch der im nationalen und v. a. internationalen Raum wie ein Gesetz gehandelten Grundannahme eines immer weiter wachsenden Energiebedarfs. Diese Grundannahme entspricht zwar der historischen Erfahrung, weil selbst in reichen Ländern der Energieverbrauch (ich vermeide hier bewusst das Wort Energiebedarf) bis in jüngste Zeit unaufhörlich gewachsen ist. Wenn man jedoch näher hinschaut, sieht man, dass die großen Energieverbrauchsschübe in Zeiten sehr niedriger Energiepreise entstanden. Und niedrige Preise sind ja kein Naturgesetz.

\section{Die technische Seite}

Faktor Fünf besteht zu zwei Dritteln aus (laienverständlichen) technischen Ausführungen des australischen Ko-Autorenteams über Einsparpotenziale in den Sektoren, die man gemeinhin für energetisch unverbesserlich hält: Schwerindustrie, Landwirtschaft und Verkehr. Nur eines der vier Sektorenkapitel beschreibt einen Bereich, der auch in der deutschen Diskussion als sehr aussichtsreich für die Verbesserung der Energieeffizienz gilt: die Gebäude. Daneben bietet das Buch über eine Website analoge Untersuchungen für Gaststätten, Supermärkte, Informationstechnik und Papierwirtschaft an. Die durchgängige Botschaft ist: Ein Faktor Fünf in der Verbesse- 
rung der Energieeffizienz ohne Leistungsverzicht ist technisch gesehen allemal drin.

Bei Gebäuden steht das von Wolfgang Feist in Darmstadt entwickelte „Passivhaus“ mit erstklassiger Isolierung und Wärmerückgewinnung mit Einsparungen von etwa 90 Prozent der Heizenergie im Vordergrund. Und inzwischen liegt das Hauptgewicht auf der Altbausanierung, wo immer noch 85 Prozent Effizienz gewonnen werden kann. Alles in allem ein mächtiges Beschäftigungsprogramm für Handwerk und Industrie. In China (Guangzhou) wurde 2010 der erste klimaneutrale Wolkenkratzer eingeweiht; auch dieser wird in Faktor Fünf porträtiert. Das Buch ist 2010 auch auf Chinesisch erschienen, weil ja China die weltweit wichtigste Baustelle für Energieeffizienz ist.

Für die Schwerindustrie werden beispielhaft Stahl und Zement behandelt. In beiden Fällen ist eine Verfünffachung der Energieeffizienz gegenüber dem heutigen chinesischen Standard machbar; 50 Prozent der Weltzementproduktion ist schließlich in China. Beim Zement geht es v. a. um den systematischen Einsatz von Geopolymeren als Ausgangsmaterial, das sind z. B. Flugasche, Hüttensand, Vulkanasche oder abbaubares Kaolin. Das erlaubt eine dramatische Absenkung der Reaktionstemperatur. In einigen Fällen geht es sogar bei Zimmertemperatur, was schon die alten Römer wussten, die ihr „opus cementitium“ für den Bau des Kolosseums oder der Trierer Porta Nigra einsetzten. Auch die Wiederverwendung von Beton aus Straßen, Mauern und Gebäuden senkt den Energiebedarf. Beim Stahl steht natürlich der Elektrostahl im Vordergrund, der in Deutschland die Siemens-Martin-Öfen längst abgelöst hat, aber wiederum in China noch die Ausnahme ist. Und in diesem Zusammenhang geht es (wie erst recht bei Nichteisenmetallen) um die systematische Erhöhung der Recyclingrate. Aber auch Endformguss, Wärmerückgewinnung, CCS und erneuerbare Energien können die Klimaverträglichkeit von Stahl verbessern.

Die Landwirtschaft war vor 200 Jahren zusammen mit den Forsten die Hauptenergiequelle aller Länder. Heute ist sie einer der größten Energieverbraucher. In die Herstellung einer Kalorie Rindfleisch werden heute bis zu 20 Kalorien Fremdenergie investiert, vom Futter aus Übersee über die Düngemittel, für Stallenergie und allerlei Landmaschinen und am Ende Schlachthof, Kühlkette und jede Menge LKW-Fahrten. Auch hier kann an allen Ecken und Enden logistisch oder technisch Energie gespart werden. Veränderte Ernährungsgewohnheiten können ebenfalls große Energie- und Treibhausgas-Einsparungen mit sich bringen.

Der Verkehrssektor gilt bei Klimaschützern oft als hoffnungsloser Fall. Das muss nicht so bleiben. Die Ausweitung des Straßengüterverkehrs war eine Folge der Just-in-time-Logistik, die in dieser Form auf billigen Tonnenkilometern basierte. Und der stetig anschwellende PKWVerkehr ging ebenfalls, angefeuert durch preiswerten Sprit, Hand in Hand mit der Zersiedlung der Landschaft. Der Ausbau des Schienennetzes und die Technologie des Umschlags zwischen Schiene und Straße waren jahrzehntelang Stiefkinder der Infrastruktur-Investitionen, und zwar weltweit, immer unter der stillschweigenden Annahme, dass die jeweiligen Marktpreise für Öl langfristig zu halten seien. Stattdessen waren, vom Leithammel Amerika ausgehend, Flug- und Straßenverkehr die Lieblingskinder privater und öffentlicher Investitionen. Faktor Fünf zeigt, dass Kombinationen von Effizienz bei Einzelfahrzeugen, Infrastruktur, Logistik und Verhaltensänderungen (z. B. Telearbeit) die nötigen Effizienzverbesserungen ermöglichen.

\section{Die politische Seite}

Die Transformation in Richtung eines Faktors Fünf mag langfristig auch vom Markt in Gang gesetzt werden. Jedoch ist der Markt in der Vergangenheit stets mit dem „Rebound-Effekt" verbunden gewesen: Alle Effizienzgewinne sind von zusätzlichem Konsum ,aufgefressen“, also überkompensiert worden. Das lag wohl hauptsächlich daran, dass die Effizienzfortschritte, wie sie seit der Mitte der 1970er Jahre weltweit erzielt worden sind, auch dazu beigetragen haben, dass die Ölnachfrage etwa ab 1982 hinter dem steigenden Ölangebot zurückblieb und ein dramatischer Preisverfall stattfand, welcher seinerseits dann den Verbrauch wieder rasant ankurbelte.

Die Märkte sind nicht geeignet, eine dauerhaft nachhaltige Entwicklung der Technologie 
und des Verhaltens herbeizuführen. Die öffentliche Hand, und damit die Politik, muss diesen Prozess steuern. Mit Abstand am wichtigsten scheint mir eine Politik, bei welcher man die Energiepreise aktiv staatlich beeinflusst im Sinne einer systematischen Anhebung. Wenn also auf den Märkten die Preise purzeln, würde der Staat aktiv gegensteuern (und dabei als angenehmen Nebeneffekt unverhoffte Steuereinnahmen erhalten). Teil des Schemas sollte es aber auch sein, auf dem Markt entstehende Preisspitzen zu korrigieren, diesmal nach unten. Das Ziel ist ein für Investoren und Verbraucher verlässlicher Preispfad, der zu langfristigen Effizienzinvestitionen im Kapitalstock, also Technologie, Infrastruktur, Fabriken und Gebäuden ermutigt.

Vorbild ist, wie bereits betont, die dynamische Entwicklung der Arbeitsproduktivität, die ja im Laufe der Industriellen Revolution seit etwa 180 Jahren um den Faktor 20 zugenommen hat, mit der Tendenz zur Beschleunigung. Doch diese Steigerung ist, wie man weiß, mit einer praktisch gleichgroßen Steigerung der Bruttolohnkosten einhergegangen. Bruttolöhne und Arbeitsproduktivität haben sich als Paar gegenseitig hoch geschaukelt. Für die Erhöhung der Löhne bedurfte es allerdings keines Staatseingriffs, weil die Arbeitnehmer sie in immer neuen Auseinandersetzungen erkämpft hatten. Kilowattstunden und Ölfässer kämpfen jedoch nicht um höhere Bezahlung. Im Laufe der letzten 200 Jahre haben die Primärrohstoff- und Energiekosten laufend abgenommen, von kurzzeitigen Preisspitzen wie 1973 und 2007 einmal abgesehen ${ }^{1}$. Aus der Dynamik mit der Arbeitsproduktivität kann man versuchsweise schlussfolgern, dass man ohne Schaden für die Wirtschaft auch die Energie- und Materialpreise in dem Maße anheben dürfte, wie die Effizienz im Vorjahr zugenommen hat; das Kalenderjahr ist ja etwa die zeitliche Schrittgröße von Tarifverhandlungen.

Ein solcher politisch festzulegender Pfad hätte das Potenzial, zu einer laufenden Beschleunigung der Produktivitätsentwicklung zu führen. Denn Investoren und Entwicklungsabteilungen der Firmen haben ja großes Interesse an der Antizipation künftiger Geschehnisse. Ich sehe technisch keinerlei Grund, warum nicht auf Dauer ein Faktor 20 erreichbar sein soll.
Es gibt immer noch ein Problem der Sozialpolitik und der Strukturpolitik. Technische Effizienzverbesserungen kommen tendenziell bei den wohlhabenden Schichten und bei den modernen Branchen früher an als bei sozial schwachen Familien und bei althergebrachten Branchen. Härten kann man dann mit klassischen Mitteln der Sozial- und Strukturpolitik abfedern. Man darf nur nicht so weit gehen, den Ausbau energetisch ineffizienter Produktion oder unnötigen Konsums zu subventionieren.

Wenn es politisch gelingt, einen solchen Preispfad festzulegen, dann machen auch ordnungsrechtliche Begleitmaßnahmen Sinn, wie Gebäude-, Fahrzeug- und Maschinen-Effizienzstandards. Eingebettet in den Preispfad sind sie nämlich nicht mehr in Gefahr, einfach den Rebound-Effekt zu verstärken.

Die Wettbewerbsfähigkeit der Wirtschaft sollte durch eine ökologische Preispolitik gestärkt und nicht etwa geschwächt werden. Anders als bei den in unangenehmer Erinnerung gebliebenen Hochpreisphasen der späten 1970er Jahre sowie des jetzt zu Ende gehenden Jahrzehnts flösse ja nicht Volksvermögen in Multimilliardenhöhe in die Ölförderländer $a b$. Und die Energieeffizienz wird ohnehin mehr und mehr zu einem der wichtigsten Wettbewerbsfaktoren.

\section{Technische Einwände, die keine sind}

Gegen die hier geäußerte optimistische Perspektive einer langfristigen Erhöhung der Ressourcenproduktivität kommen immer wieder Einwände aus Technik und Naturwissenschaft. So argumentiert die energieintensive Industrie im politischen Raum stets damit, dass ihre Einzelprozesse eine weitere Steigerung der Energieproduktivität gar nicht mehr zulassen, weil sie bereits am ,thermodynamischen Anschlagpunkt" sind. So ist natürlich das Schmelzen von Aluminium aus Bauxit ständig energetisch optimiert worden, mit immer geringeren Effizienzgewinnen, weil man an physikalische Grenzen stößt, die man mit noch so schmerzhaften Preisstrafen nicht überwinden kann. Ähnliches gilt von der Chloralkalielektrolyse oder der Herstellung von Portlandzement. 
Die Antwort darauf sollte aber nicht der verbilligte Bezug von Energie sein. Man muss differenziert antworten. Bei Aluminium etwa ginge es um eine weitere Steigerung des Recyclings, um die Verlagerung der Primärschmelze in Länder mit natürlichen billigen Energiequellen und die Substitution des Metalls durch funktional ähnliche Stoffe wie Hartholz oder gewichtsreduzierte Eisengitterstäbe. Beim Zement wird gern behauptet, dass die Alternativen zum Ausgangsstoff Kalkstein (der die hohen Temperaturen benötigt), längst ausgeschöpft seien, was aber nicht zutrifft (Achternbosch et al. i. E.). Bei der Chloralkalielektrolyse ist die wichtigste Antwort die Substitution von PVC durch Polyethylen. Und bei vielen für Hochtechnologie benötigten Metallen liegen die heutigen Recyclingraten unterhalb von einem Prozent (Graedel et al. 2011/i. E.). Hier sind also noch gewaltige Verbesserungen erreichbar, sobald diese rentabel erscheinen. Gewiss ist in manchen Bereichen eine internationale Koordinierung der Politik wünschenswert, um bloße geographische Wanderungen der Industrie zu vermeiden. Aber ein gewisses Maß der internationalen Arbeitsteilung nach Gesichtspunkten von Bevölkerungsdichte, technischen Potenzialen und geologischen Bedingungen ist nicht vermeidbar und auch nicht schädlich. Eine Deindustrialisierung nach dem mit Recht beklagten Modell von Großbritannien ist mit dieser Aussage in keiner Weise impliziert. Japan, Südkorea und sogar China haben sich auf den Weg gemacht, die Industrien und Technologien des frühen 20. Jahrhunderts auszulagern - um ihrer internen Wohlstandsentwicklung willen.

Mit einer zunehmenden Informationsintensität der Wertschöpfung und dem Auftauchen von eleganten Nanotechnologien scheint die Welt ganz unabhängig von den Klimabesorgnissen auf einen Pfad eingeschwenkt zu sein, der die Energieproduktivität steigen lässt. Substitution von knapp gewordenen oder zu aufwändig gewordenen Grundstoffen war seit alters her ein Merkmal des Strukturwandels und nicht etwa etwas Anstößiges. Auch die Infrastruktur und die täglichen Gewohnheiten haben sich immer wieder neu ausgerichtet. Diese normale Entwicklung in der richtigen Richtung, aber sozialverträglich zu beschleunigen, ist ein legitimes Ziel der Politik. In einem Hochtechnologieland wie Deutschland sind Naturwissenschaft und Technik in besonderem Maße gefordert, diese Entwicklung kreativ zu begleiten und nicht etwa durch Verweise auf angebliche technische Barrieren zu behindern.

\section{Anmerkung}

1) Vgl. Abbildung 9.1 in Faktor Fünf, basierend auf Zahlen des Bank Credit Analyst.

\section{Literatur}

Achternbosch, M.; Kupsch, Chr.; Nieke, E.; Sardemann, G., i. E.: Gegenwärtige und zukünftige Chancen der globalen Zementindustrie zur Reduktion ihrer $\mathrm{CO}_{2}$-Emissionen. In: GAIA 2011, i. E.

BMWi-Bundesministerium für Wirtschaft und Technologie, 2010: Energiekonzept für eine umweltschonende, zuverlässige und bezahlbare Energieversorgung. Berlin

Graedel, Th. et al., 2011/i. E.: Recycling Rates of Metals. A Status Report. UNEP-DTIE, Paris

von Weizsäcker, E.U.; Hargroves, K. et al., 2010: Faktor Fünf. Die Formel zum nachhaltigen Wachstum. München

\section{Kontakt}

Prof. Dr. Dr. h.c. Ernst Ulrich von Weizsäcker

Co-Chair, International Panel for Sustainable

Resource Management

Postfach 15 47, 79305 Emmendingen

Fax: +49 (0) 7641 / 9542217

E-Mail: ernst@weizsaecker.de 